\title{
A formalization of one of the main claims of "Mutations in NGLY1 cause an inherited disorder of the endoplasmic reticulum-associated degradation pathway" by Enns et al. $2014^{1}$
}

\author{
Núria Queralt-Rosinach \\ Leiden University Medical Center, Leiden, The Netherlands \\ E-mail: N.Queralt_Rosinach@lumc.nl; ORCID: https://orcid.org/0000-0003-0169-8159 \\ Editor: Cristina-Iulia Bucur (https://orcid.org/0000-0002-7114-6459) \\ Review comments from: Tobias Kuhn (https://orcid.org/0000-0002-1267-0234); Cristina-Iulia Bucur \\ (https://orcid.org/0000-0002-7114-6459)
}

Received 3 June 2021

Accepted 17 December 2021

\begin{abstract}
Enns et al. claimed in previous work that NGLY1 deficiency is a novel autosomal recessive disorder of the ERAD pathway. We present here a formalization of that claim, stating that all things of class "NGLY1 deficiency" that are in the context of a thing of class "human" always have a relation of type "is caused by" to a thing of class "dysfunction of ERAD pathway" in the same context.
\end{abstract}

Keywords: Human, NGLY1 deficiency, dysfunction of ERAD pathway

\section{Introduction}

Enns et al. [2] state that "NGLY1 deficiency is a novel autosomal recessive disorder of the endoplasmic reticulum-associated degradation pathway.". We present here a formalization of the main scientific claim from this quote by using a semantic template called the super-pattern [1].

\section{Formalization}

Our formalization looks as follows:

\footnotetext{
${ }^{1}$ As RDF/nanopublication: http://purl.org/np/RA12lVwEtmddK9OwDkZQZlgJaOD2-0NXtAtO_jDaG-3VQ
} 


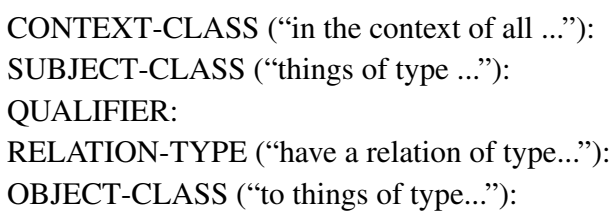

\author{
human \\ NGLY1 deficiency \\ always \\ is caused by \\ dysfunction of ERAD pathway
}

In the context class we use the class "human" (Q5) from Wikidata. In the subject class, we use the class "NGLY1 deficiency" from Mondo Disease Ontology. In the object class we minted a new class "dysfunction of ERAD pathway" that is related to the class "ERAD pathway" (Q21101062) from Wikidata.

\section{RDF code}

This is our formalization as a nanopublication in TriG format:

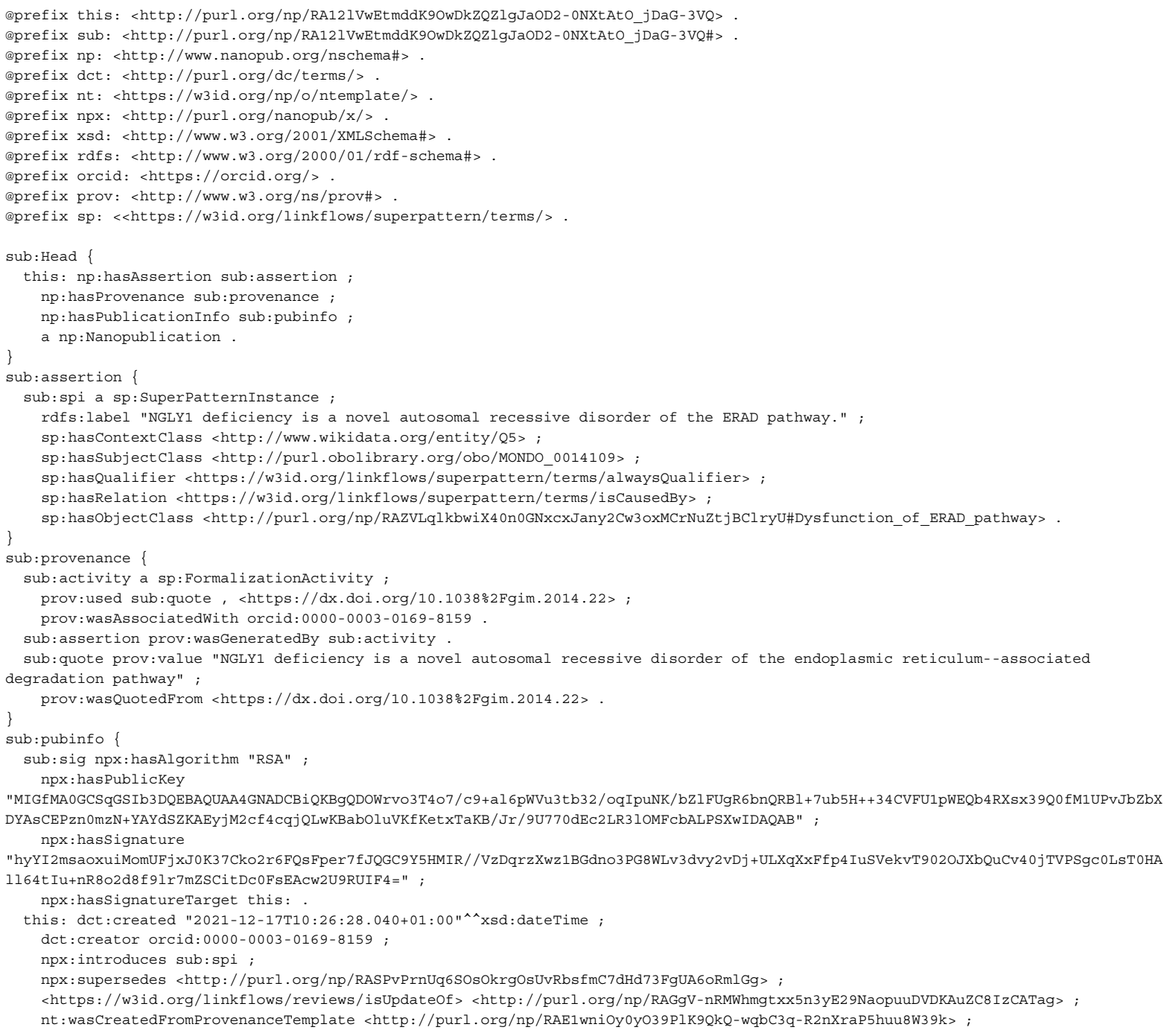


nt: wasCreatedFromPubinfoTemplate <http://purl.org/np/RA2vCBXZf-icECVRGhulJXugTGxpsV5yVr9yqCI1boh4A> , <http://purl.org/np/RAA2MfqdBCzmz9yVWj KLXNbyfBNCWSMmOqCNUxkk1maIM> <http://purl .org/np/RAjpBMlw3owYhJUBo3DtsuDIXsNAJ8cnGeWAutDVjuAuI> ;

nt:wasCreatedFromTemplate <http://purl.org/np/RAv68imZrEjfcp2rnEg1hzoBqEVc0cQMtp9 1Za0BxNM4 > .

The following nanopublications introduce the newly minted classes in TriG format.

This is the class definition of "dysfunction of ERAD pathway":

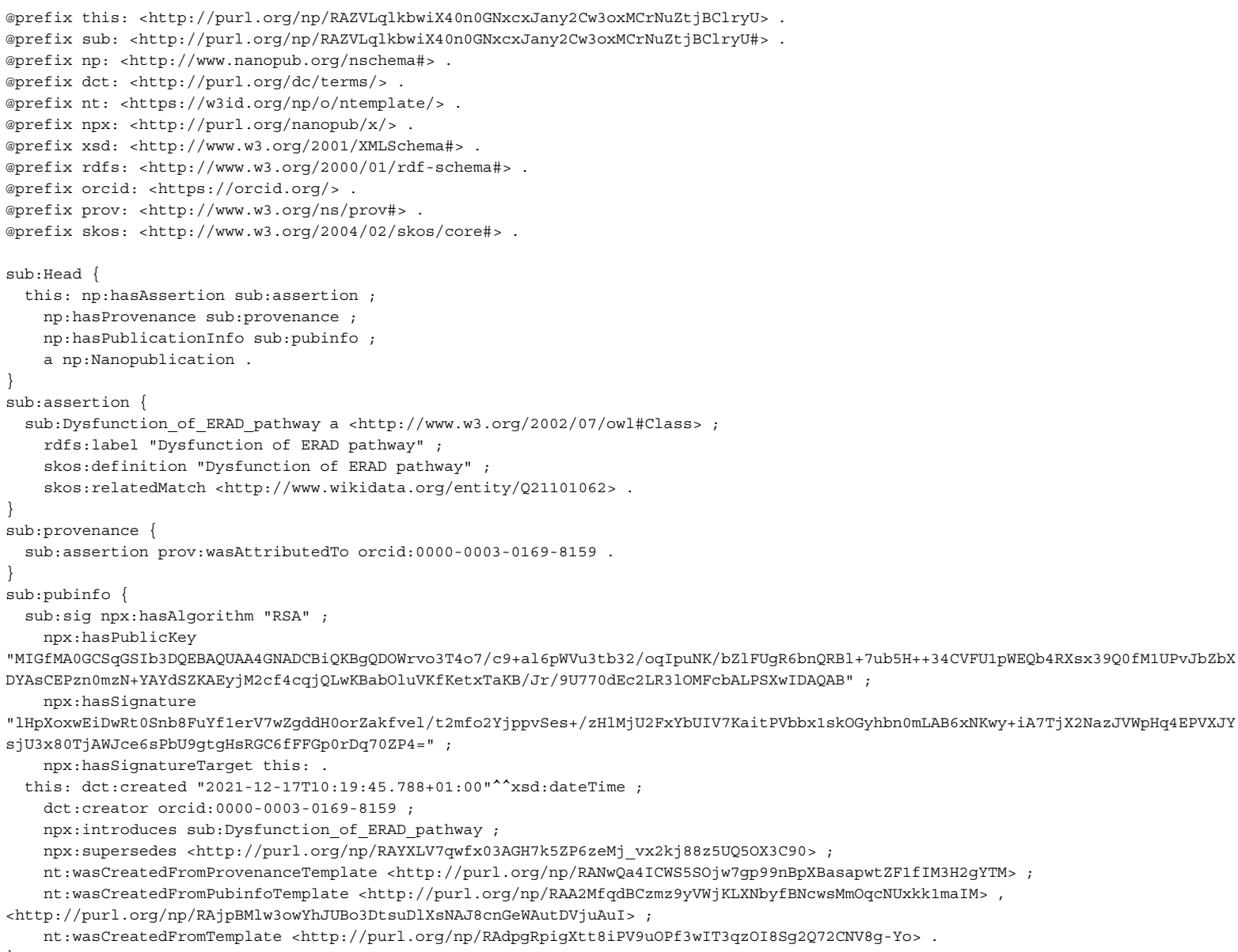

\section{References}

[1] C.I. Bucur, T. Kuhn, D. Ceolin and J. van Ossenbruggen, Expressing high-level scientific claims with formal semantics, in: Proceedings of the 11th Knowledge Capture Conference, 2021. doi:10.1145/3460210.3493561.

[2] G. Enns, V. Shashi, M. Bainbridge et al., Mutations in NGLY1 cause an inherited disorder of the endoplasmic reticulumassociated degradation pathway, Genet Med 16 (2014), 751-758. doi:10.1038/gim.2014.22. 